\title{
Editorial
}

\section{OPERATIONAL PROBLEMS IN LEPROSY PROGRAMMES WHEN THE ENDEMICITY DECLINES}

In many leprosy endemic countries, the large scale application of dapsone monotherapy has been followed by a marked reduction in prevalence, but by a less marked and only gradual reduction in the case detection rate, which is the only measure of incidence available to us. It is probable that factors other than control by chemotherapy have played a part in this decline. Be that as it may, there is no doubt that the correct application of multidrug therapy (MDT) will, either directly or indirectly, have a rapid and dramatic effect in reducing the caseload in many leprosy control programmes. A very large number of cases will be assessed and discharged before MDT is introduced and this will be followed by the discharge from treatment of a high proportion of the cases of paucibacillary leprosy within the first year. There are likely to be many other changes of a varying nature and these can perhaps be divided into two groups, administrative and epidemiological.

\section{Administrative changes}

Based on long experience of the management of leprosy control projects, a Leprosy Mission International study group ${ }^{1}$ has made the following realistic assessment of the probable effects of MDT programmes of average to low efficiency:

The case load will be reduced by $50 \%$ in 5 years.

2 Thereafter the intake of new cases will remain fairly stable and maintain the level of the case load for several years, falling only gradually-provided that similar measures are being applied in adjacent areas and that the number of new cases is not inflated by immigrants.

In programmes where the level of efficiency may be higher, the caseload reduction may be considerably greater: for instance, in Malawii, ${ }^{7}$ in the 5-year period 19801984 there has been a reduction from nearly 19,000 in 1980 to just over 5000 in 1984. 


\section{Epidemiological changes}

Pointing out that there is, for several reasons, likely to be a decline in incidence rates in many leprosy endemic countries, Irgens ${ }^{2}$ has analysed data from Norway and from several other countries where such a decline has already occurred. He draws attention to two changes in the general pattern associated with the long incubation period of many leprosy cases:

There is a shift towards the older age groups among the newly detected cases. In most countries (India being the exception) there is an increase in the lepromatous proportion among newly detected cases.

Other workers have reported important differences, having practical implications for the organization of a control programme:

In India, in areas of low endemicity, the proportion of household infections is higher than in areas of high endemicity. ${ }^{3}$

In the Philippines, although there has been a decline in prevalence, the numbers of new cases have not declined to the same extent. There has, however, been a gradual regular reduction in the case detection rate. ${ }^{4}$

In many South American countries, on the other hand, with the exception of Venezuela, the decline in prevalence has not been accompanied by any reduction in incidence. ${ }^{5}$

With this background of data and expert opinion, we can now proceed to discuss the operational problems likely to beset leprosy control programmes in the future. These may be considered under the following headings:

1 The need to maintain a high standard of early diagnosis.

2 This is largely dependent on the maintenance of a degree of priority at national (and local) levels for the provision of adequate leprosy treatment, training and health education.

3 The need for periodic review of referral facilities and the care and treatment of complications.

4 The proper employment of staff with specialized training in leprosy, who will be underutilized as their caseload is reduced.

The relative importance of these four considerations is likely to vary depending in particular on whether the programme is organized vertically or is integrated with the general health service but it is probable that all will apply to some degree to all programmes.

\section{THE NEED TO MAINTAIN A HIGH STANDARD OF EARLY DIAGNOSIS}

There is a general impression - though, as far as we know, no firm evidence-that the 'Index of Suspicion' both among the public and among the staff of the basic health services is lower in areas of low prevalence. This is certainly well known in 
the case of tuberculosis. ${ }^{6}$ The less leprosy there is in the community, the less likely are members of the family and other close associates to suspect leprosy in one of their number, and similarly, the staff of the basic health unit are less likely to think of the possibility of this diagnosis. In addition, it is only to be expected that the less one encounters leprosy, the less practice one has in the clinical skills required to make the diagnosis. Although not invariable and universal, there is often a relative increase in the proportion of new cases with multibacillary leprosy, the early forms of which are often more difficult to diagnose clinically and require confirmation by skin smear, so that delay in the diagnosis of new cases is a very real danger. Continuing medical education for health staff and health education for the public are vital.

THE NEED TO MAINTAIN A DEGREE OF PRIORITY AT NATIONAL (AND LOCAL) LEVELS FOR PROVISION OF ADEQUATE LEPROSY TREATMENT, TRAINING AND FOR HEALTH EDUCATION

It will be a great temptation to assume that a decline in the overall caseload, the registered prevalence, indicates a decline in incidence and a decline in the public health importance of leprosy. As we have seen this is not so, and it requires many years of patient, painstaking work for control by chemotherapy to have an effect on the transmission of the disease. It is essential, therefore, to emphasize the importance of maintaining: (a) adequate resources in terms of personnel, drugs and other equipment in order to treat all existing and new leprosy cases; (b) an appropriate level of orientation in leprosy in the training of health personnel of all cadres and continuing refresher training for specialist staff, especially those responsible for supervision and laboratory services; (c) programmes for health education about leprosy for the general public; and (d) ensuring national policy on the above aspects is implemented at local level.

\section{THE NEED FOR PERIODIC REVIEW OF REFERRAL FACILITIES AND THE CARE AND TREATMENT OF COMPLICATIONS}

It can be deduced from the data quoted above that the numbers of patients with complications will not fall to the same extent as the total numbers under treatment. Because of delay in diagnosis it is likely that the proportion with established disability at the time of initiation of treatment will increase. The discharge from treatment of a high proportion of patients with paucibacillary leprosy will result in the accumulation of multibacillary leprosy cases on the treatment registers and possibly these may be augmented by a disproportionate number of new cases. Consequently one can expect, firstly, that the numbers of cases requiring monitoring of nerve function deficit and the preventive care of disabilities will not decrease to the same extent as the total caseload and, 
secondly, that the numbers requiring specialized treatment for reaction, both reversal reaction and ENL, will remain fairly stable. In addition, the patients who are no longer under chemotherapy but who have disability will continue to need care and regular follow up, including replacement footwear, surgery and physiotherapy.

THE PROPER EMPLOYMENT OF STAFF WITH SPECIALIZED TRAINING IN LEPROSY WHO WILL BE UNDERUTILIZED AS THEIR CASELOAD IS REDUCED

Vertical programmes employing staff trained only for the diagnosis and treatment of leprosy will have to arrange for their gradual retraining and redeployment and for the integration of their duties into the general health service. This may not always be easy. There will, however, continue to be a need for specialized supervision for the reasons already cited. No doubt the quality of the service could be improved by the supervisors spending some of the time available on more informal teaching of the staff of the basic health units visited, more attention to the taking and recording of skin smears, to the monitoring of nerve function deficit and the preventive care of disabilities, and on the compilation of case records and statistics. It is probable, however, that many programmes will face strong arguments questioning whether this specialized kind of supervision is economically justifiable, and in these cases it may only be feasible if combined with other duties. In some circumstances this can be done by basing the supervisor at a Health Centre; elsewhere it may be possible to make use of his special skills in fields such as the following:

In the field of Chronic Communicable Disease Control: pulmonary tuberculosis (experience in combined TB/Leprosy Control Programmes is encouraging); trachoma; general health surveys; health education duties; and in the future, in diseases for which 'pulse' therapy may become available, e.g. onchocerciases.

2 In the care and referral of disabilities, as in leprosy cases: orthopaedic problems, such as post poliomyelitis paralysis, amputations etc., requiring firstly, referral for surgery and/or orthopaedic appliances and secondly, regular follow up; ophthalmic problems, in which all leprosy staff should have had both training and experience, firstly in the first aid field treatment of minor conditions and secondly in referral to the ophthalmic specialist.

3 In the treatment of other chronic medical conditions requiring long term regular medication and periodic supervision, which basic health services often have difficulty in supplying at village level, for example: diabetes; and certain psychiatric disorders.

We should, therefore, look to the future with optimism. Our leprosy control programmes will almost certainly cater for fewer numbers, but there must be no lowering of the standards or input from the donor agencies and, where feasible, 
the staff and the facilities until now available only for leprosy sufferers, can be used to meet other needs also.

\section{Upland Road}

Sutton, Surrey SM2 5JE

H W WHEATE

Lepra, Fairfax House,

G F HARRIS

Causton Road, Colchester COI IPU

\section{References}

1 Askew AD. Managerial implications of Multidrug Therapy. Lepr Rev, 1985; 56: 89-97.

2 Irgens LM. Secular trends in leprosy: increase in age at onset associated with declining rates and long incubation periods. Int J Lepr, 1985; 53: 610-17.

${ }^{3}$ Christian M. The epidemiological situation of leprosy in India. Lepr Rev 1981; 52: Supplement I, 35-41.

${ }^{4}$ Ito T. The epidemiological situation in South East Asia. Ibid. 43-51.

${ }_{5}$ Motta CP. The epidemiological situation in the Americas. Ibid. 61-8.

${ }^{6}$ Horne NW. Problems of tuberculosis in decline. Editorial, Brit Med J 1984; 288: 1249-50.

7 Lepra in Malawii. Annual Report 1984. Lepra (British Leprosy Relief Association), Fairfax House, Causton Road, Colchester COI 1 PU, England. 